\title{
Can speed enhance our understanding of the role of spatial connectivity? The creation of a 'Spatial-Speed' map
}

\author{
Paul Goodship \\ Bartlett School of Architecture, University College London. UK \\ E-mail: ucftpgg@live.ucl.ac.uk
}

\begin{abstract}
Throughout Latin America urban cable-cars have fast become a normal sight with urban transport systems, taking residents and tourists to and from previously isolated locations. As the popularity of these new modes of transport grows, it is important to understand the role spatial connectivity plays in integrating previously segregated communities. This is possible using a Space Syntax methodology to analyze the connectivity of a spatial network. However, this does not take into account different forms of movement affected by transport or local landscape. Therefore, the aim of this paper to explores the use of 'speed' as a measurement to enhance our interpretation of spatial connectivity, through the case of Medellin. 'Speed' is used because it provides a clear indication of connection times between different parts of the city and is comparable throughout a variety of conditions, such as transport and walking. An average speed is therefore calculated for each segment of Medellin's spatial network, including all forms of transport, and is then combined with the results of a standard Space Syntax analysis, forming a hybrid 'spatial' and 'speed' map. For accuracy, the results are tested against a pedestrian movement survey conducted locally nearby each cable-car station. The findings indicate that by introducing 'speed' as a weighted measurement, the overall spatial network of the city is not significantly improved, yet when the area surrounding each cable-car is examined closely, local 'through'spaces is clearer, especially when spatial conditions, or the user, is nonstandard.
\end{abstract}

Keywords: Space Syntax, Speed, Spatial Connectivity; Medellin.

\section{Medellin and The Urban Cable-Car}

Medellin the second largest city in Colombia has recently become one of the most talked about cities in the world, due to its rapid transformation from a violent, drug cartel controlled city to a city that aspires to be more socially inclusive. This transformation has been encapsulated by new forms of urbanism that aim to engage the poorest communities and a municipality that inspires to integrate all of its citizens into one city. The most important urban upgrading project used to develop this was 'Proyecto Urbano Integral' (PUI) ${ }^{1}$ and this aims to increase the quality of life for inhabitants, focusing on areas where poverty and violence is most visible. Its strategy focuses on an integral and comprehensive approach, providing each project with three main components - a physical, social and institutional component. Yet, one of the most essential elements to its initial success was the newly opened urban cable-car, which connected the previously isolated neighbourhoods and this intriguingly was not initially a part of the program. However, it quickly became more than just a simple transport connection, it enabled the upgrading projects to become a part of the citywide transformation process and opened up the neighbourhood (and the realities within) to all citizens of Medellin. This saw major changes occurring in some of the most violent and poor neighbourhoods of Medellin and with it much international acclaim. 
This brings to the forefront the importance of the urban cable-car in this upgrading programs and allows us to speculate the important role of spatial connectivity in the overall transformation process of Medellin's informal settlements. In the past this has been explored using Space Syntax (Goodship, 2015a) (Goodship, 2015b). Here the spatial analysis of the city reflects many of the standards patterns that would be expected. As the informal settlements showing a high level of centrality when a anaysed at the local scale, then the city centre shows a higher levels of integration at a larger scale and the most important 'through' routes are picked for local urban centres. Then when the cable-car is introduced to the analysis the impact, while very small, is picked up and shows where the cable-car connects best with the rest of the city, thus allowing the local urban centres surrounding each station to have transform differently.

However, the locations of the cable-car lines are on very steep slopes and when the results of this analysis are examined more closely, specifically at a local scale, (Figure 1) it becomes apparent that the analysis does not pick up elements that may hinder movement, such as steepness or poor road conditions. The steepness and complexity of the locations of each cable-car line can be easily understood in 3-dimenstions and with the contour map of the city. This is essentially why the cable-car transport system became so successful, since it could traverse up and down the steep valley faces unhindered. From this brief overview it is easy to appreciate why the steepness of certain streets may influence forms of natural movement in and around each cable-car station. So while Space Syntax has a tradition of accurately recording movement, via its 'to' and 'through' space measurements, it is possible that it's $2 \mathrm{~d}$ dimensional method of measuring space may not pick these types of local variables that affect local movement.

\section{Local Movement}

In order to understand how the local landscape affects local movement, a series of walking exercises where performed around each of the six cable-car stations (not the interchange stations), each roughly the same length and time. These exercises used GPS to record the elevation above sea level and the time and length of each walk so that an average speed can be calculated. While these measurements are associated solely to the author of this paper and therefore should be used as a valid indicator for a whole community, it does provide some basic movement evidence to confirm the influence the local landscape has of movement.

This shows that local movement and landscape is very similar for all the stations, apart from La Aurora. As the average walking pace for these five stations are between $3.28-$ $3.67 \mathrm{~km} /$ per hour, which is significantly slower than the average pace of $5 \mathrm{~km} /$ per hour on flat land and at La Aurora the average speed is $4.42 \mathrm{~km} /$ per hour. This is also reflected in the average elevation height of each site, as on average the difference between the highest and lowest points of each walk is between 160 and 210 metres, whereas at La Aurora this is only $116 \mathrm{~m}$. This unsurprisingly demonstrates that the flatter the land the easier it is to move freely. While it is hard to use this type of data to develop scientific findings, as only one participate is used, these basic statistics are enough to make it apparent that the landscape is effecting movement differently around each station.

While it is evident that the local landscape is affecting movement, what is also clear is that public transport is connecting citizens to different spaces in the city at different speeds. This is important because the spatial connections of both the cable-car and the overground train system are included as equal connections within the spatial analysis of the previously discussed research.

The differences in transport speeds can be recorded in the same way as the walking observations. The average speed of the cablecar is recorded between 10.91 and $12.85 \mathrm{~km} /$ hour and the average speed of the overground train is between 31.79 and $33.33 \mathrm{~km} /$ hour. These are the average speeds recorded for the full length of each line, thus there are points along the line where the train travels faster or slower and during rush hour the time spent at each station maybe longer. Then when 15 different local bus routes (not the BRT) are recorded the average speed was $16.52 \mathrm{~km} /$ hour. 

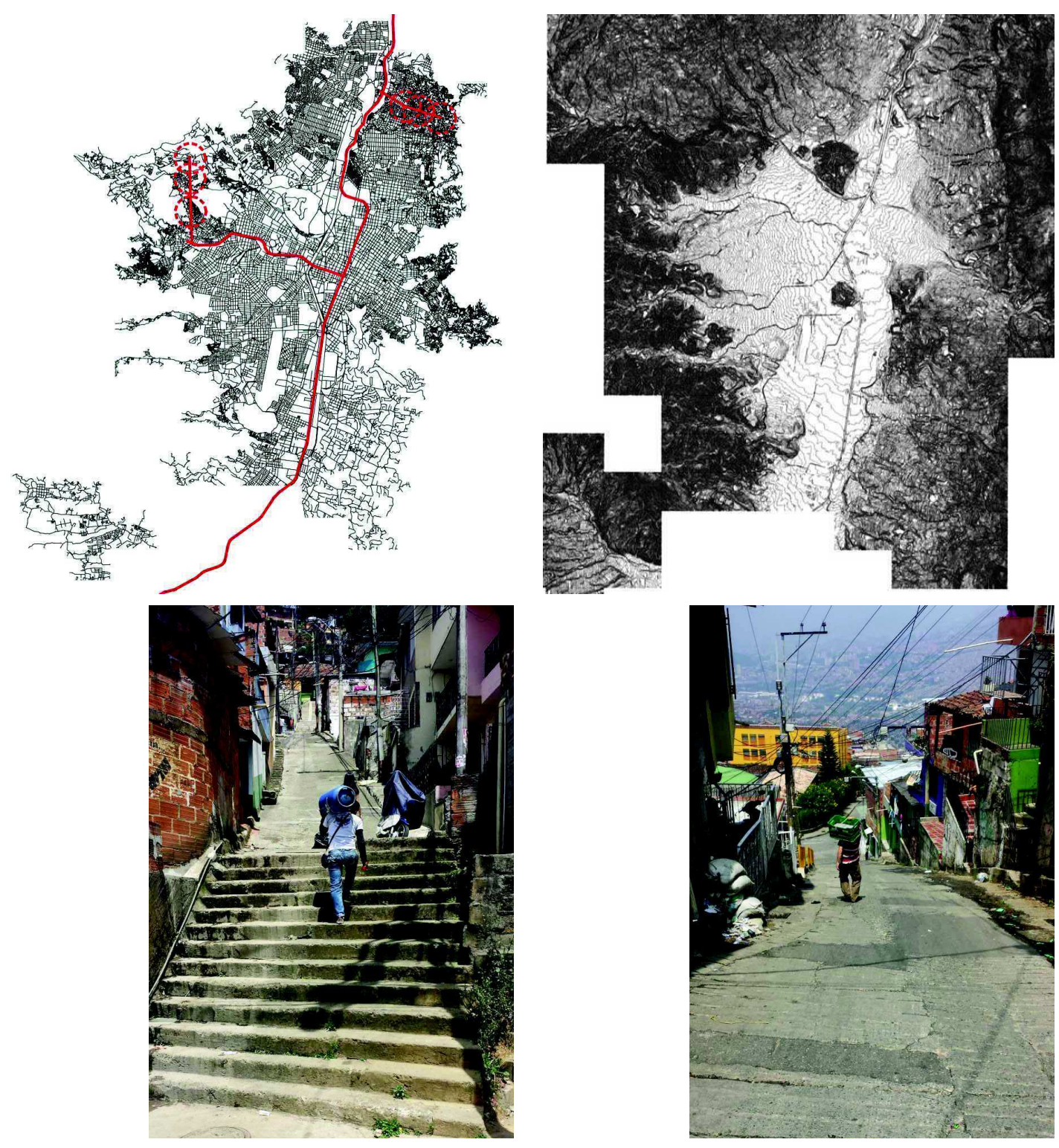

Figure 1.

Local Landscape. A) Medellin Urban Grid. Red line denotes Metro transport system. Dotted red circles denotes Cable-car stations. B) Medellin contour map. C \& D) Calle 105 in Santo Domingo, a typical steep street found in an informal settlement

What these results clearly demonstrate is the way different spaces in the city are connected by alternative forms of transport (not including private vehicles) and how the connectivity of each is comparable by speed. For example, if someone used the Metro system (both the cablecar and overground train) to go from the Santo Domingo to the San Antonio in the city centre, this would take 22.5 minutes and using a bus to travel to the same destination would takes 30 minutes, though this can be heavily affected by traffic. Then to walk between Acevedo and
Santo Domingo this takes around 45 minutes because it is up the slope, whereas the opposite directions is much shorted at 30 minutes. Understandably, connections between similar spaces vary significantly between different forms of transport and landscape. While this is very obvious, in cities like Medellin movability is very important when understanding the role of spatial connectivity, since these significantly affect the way different spatial connection (Figure 2). 

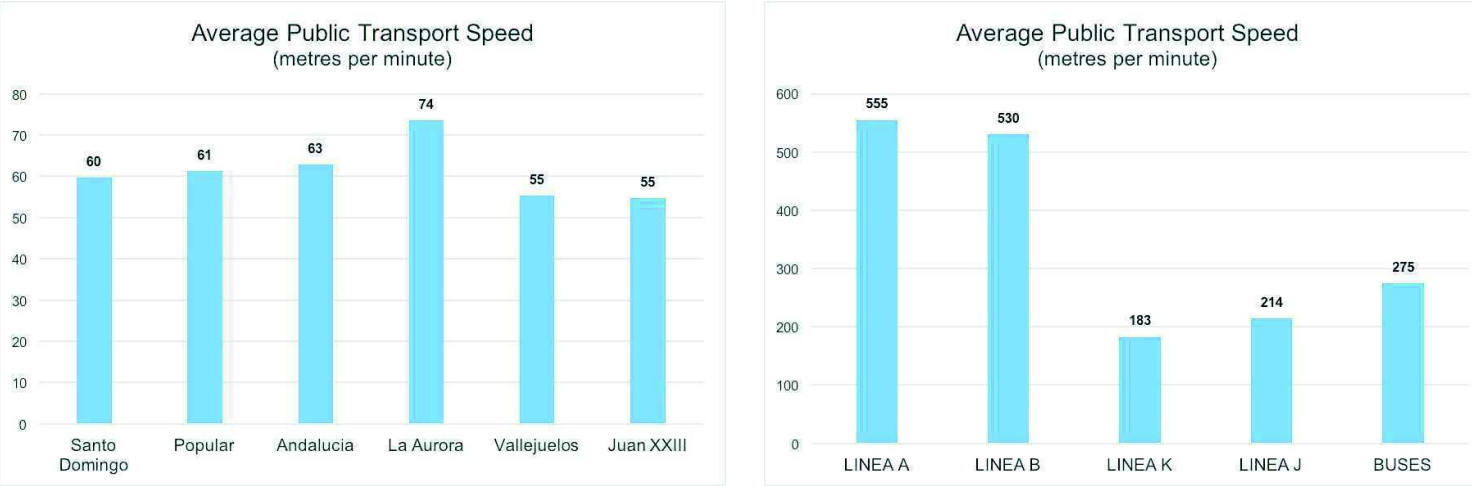

Figure 2.

Varying Movement Speed. Left - Average walking speeds within surrounding areas of each cable-car station. Right - Average public transport speeds in Medellin

\section{Spatial Connectivity}

This bring into question the use of a twodimensional spatial analysis technique to measure the impact, since local conditions are clearly influencing movement. So, while Space Syntax has a long established connection to urban theory that can 'link physical aspects of the urban system with its functional, social and behavioural aspects, directly and seamlessly' it could be possible that it's two dimensional restrictiveness may prevent these local conditions from being a part of the analysis (Karimi et al 2013).

As Vinicius Netto points out 'few sociospatial approaches have been quite so polemical and stirred quite so many different responses from scholars of the city as space syntax' (Netto, 2016). So whilst Space Syntax has established itself as a both a reliable methodology and a well-respected urban theory, there are still many detractors. He further explains that many cite its epistemological limitations and 'accuse the theory of excessive formalization and geometric reduction, questioning its capacity to represent the city, its morphology and transformations, and the partial way in which it captures the complexity of the social world' (Netto, 2016). This suggests that Space Syntax can be seen to create a world that is almost exclusively pure geometry and structure, to allow its analysis to focus on configuration and hierarchies. Yet, building heights change from one location to another, thus modifying movement - taller buildings acting as generators of greater movement (Ratti, 2004).
Then another commentator Stephen Read points to two commonly stated weak features, 'one is its tendency to treat the urban object as a thing bounded by the limits of the densely built fabric of the centre; the other, to treat all movement spaces equally when it is quite clear that different classes of physical space in the fabric of the city perform quite differently at the levels of urban speed and function and at the level of the human experience of space and time' (Read, 2005). Hence, while it provides 'indices associated with forecasting trip volumes, these are not based on models which stimulate processes of movement and thus do not provide methods for predicting the impact of locational changes on patterns of pedestrian flow' (Ratti, 2004). This presents the argument that Space Syntax can be seen to negate the way that urban mobilities and everyday dynamics affect the space of the city, especially at the level of the individual.

When we consider how we connect between the spaces, walking is of the most basic form of human movement. However, people do not simply overcome distances, 'they move within cities with different objectives, such as to do shopping, to socialize, to go to some particular space or only to wander. Everyone moves in his own way, with their unique mental and physics distinctive peculiarities and motivations. However, human behavior is based on a number of shared decision-making parameters and spatial accessibility restrictions imposed by the environment where it occurs.' Yet, it can be said that this dynamism and individuality is generalised within Space Syntax with the 
analysis instead the focusing on the larger object (Zampieri et al., 2009). If we do not consider movement in all its dimensions we are simplifying the reality by 'reducing the way we understand the pedestrian's behavior, disregarding important attributes like: ambiance; socialisation elements; health and rest; and aesthetic' (Zampieri et al., 2009). With this we start to understand the complexities that surround the simple act of walking from one space to another and whilst Space Syntax map these conditions depending on connectivity, for the individual there are many local factors that influence the simple act of walking between two points.

Alan Penn points out that Space Syntax as a theory 'measures how specific 'space' is constituted by its relations to all other 'spaces' in a system, while the theory consider an individual's identity to be constituted, not only by their subjective view, but also by the view of all others in the social group' (Penn, 2003). He points out that 'there is considerable interest in understanding the mechanisms at the individual level that give rise to these observed regularities in aggregate behavior' (Penn, 2003). This is because the dimension of time within which individuals experience the spatial and social environment is always changing. The time dimension brings with it the issue of metric space since individuals can only move within a relatively constrained range of speeds. Stephen Law et al further discuss how 'in an age where citizens are connected in an endless city of intertwining social, transport and communications networks, there is a need for Space Syntax analysis to look beyond the street network in exploring how different networks impact upon today's society' (Law et al., 2012). Thus arguing that 'the decrease in travel time through faster modes of transport, allows for a further accumulation of towns and the emergence of the metropolis. Underground stations emerge as multi-modal hubs with high volumes of pedestrian activity, land use intensity and specialization. They play an important role for the spatial economy and shift town centres from the original location' (Law et al., 2012). To prove this Law et al created a series of experiments to demonstrate how certain neighbourhoods in London located next to underground stations are better integrated.
This is not attempting to reinvent Space Syntax, but instead build upon what is successful and push for a for stronger integration between the traditional configurational analysis and geographical analysis from other sources, to create more dynamic analysis (Law et al., 2012).

For many critics Space Syntax is not fulfilling its potential as a method for understanding the dynamical forms of the contemporary city (Ratti, 2004). However, both Read (2005) and Netto (2016) clearly conclude that Space Syntax in its ambition is a way to understand complex effects, on the horizontally distributed social body of the city and its physical infrastructural movement. It ability to focus on and fully understand one key element of city - space - and connecting it to various sociopolitical and economic conditions has made it a very power theory in recent times. Therefore, while its neutrality in measuring space maybe seen as a weakness, it is also its greatest asset (Netto, 2016).

\section{The Objective}

To understand the role spatial connectivity plays in the transformation process of an informal settlement it is important to understand what is meant by spatial connectivity. Typically, Space Syntax measures connection points within the spatial network of the city and these can then be associated with certain local conditions. Yet, as discussed, the way each individual moves between these points varies dramatically on the mode of transport and the local landscape. In order to further explore the role of spatial connectivity this paper explores the worthiness of weighting the spatial network of a city with the dynamic measurement of 'speed'. At its purest form 'speed' represents the simplest method for measuring the connection times between of different parts of the city and can represent multi forms of movement, such as public transport and walking. Therefore, this article examines the influence that extreme topologies and unhindered transport systems have on the connectivity of a city and investigates if the variable 'speed' can improve the way spatial connections are understood. This is done through the case of Medellin, with 
a specific focus on the areas surrounding the cable-car transport system. This is because it provides an extreme topology with steep valley faces, where many informal settlements are located and offers a unique form of direct transport that floats over homes. This is not aiming to create a new complete methodology or contradict past ones, but instead explore and discuss how the dynamism of space can be better understood to allow the varying movement of individuals to be included in the spatial analysis of a city.

\section{Methodology}

In order to achieve this the paper attempt to examine a method that can combine local dynamic speed variables with the previously calculated spatial analysis results for Medellin. To do this an average 'speed' is calculated from two modes of movement - walking and public transport - for each segment of the spatial network previously developed (Goodship, 2015a). At this point it should be made clear that this does not include micro-buses, as whilst these are the most popular form of transport in Medellin they are very unreliable and difficult to record. Therefore, they are omitted for the ease of developing initial results. First the public transport speed is taken from the average speeds provided online and verified onsite using GPS and then the walking speed is calculated using the 'Tobler's Hiking Function' (Magyari-Sáska and Dombay, 2012). This uses the steepness of the landscape, the length of the walk and the average speed for a flat surface $(5 \mathrm{~km} /$ hour $)$ to calculate the time needed to walk a define route, which can then be converted into an average speed. To make this an automated process for the spatial model, a local contour map is converted into an elevation raster image (each pixel has an elevation value), this then allows the start and finish point of each segment of the spatial model to have an elevation value, then the 'Tobler's Hiking Function' can calculate the time needed to walk each individual segment in both directions. These results are then converted into a speed value and used to create an average 'speed' map of the city. These results are then weighted against the previous spatial analysis results by normalising the 'speed' and 'spatial' results separately and then combined together to create 'speed-spatial' values for each segment.

These results are then correlated to local movement in and around each cable-car station. To do this a movement survey counts the number of people moving through an imaginary gate at 16 different locations within a $500 \mathrm{~m}$ radius of each station entrance and is completed during a weekday between $5 \mathrm{pm}$ and $7 \mathrm{pm}$. The movement survey is then correlated to the spatial analysis results, both including and excluding 'speed', so that a comparison between the two set of results can be made. This should allow the merit of 'speed' as a dynamic variable to become apparent and provide clear findings to form an in-depth discussion into its use. The aim of this paper is not to provide a comprehensive and complete model but to test the hypothesis that movement speed influence spatial connectivity, so that further explorations into its use can be performed in the future.

\section{Results}

When Medellin was previously modeled using standard Space Syntax measurements, the results were generally as expected yet, when these are examined more closely it is apparent that the analysis does not pick up elements that may influence movement, such as the steepness of the landscape and different modes of transport. Hence, there is the potential failing in this analysis. Therefore, to examine these potential failings a speed map of the city is produced, taking into account the movement of walking and transport and then this is combined with the previous spatial analysis to examine if there is an improvement in the results.

From this initial speed map, it is clear that the areas located on steep slopes have the slowest average speed and the area on the flat have the fastest and while this is very predictable it does verify the methodology. Also the areas with the slowest speed are often where the largest concentration of informal settlements are located. This shows similarities with the municipality's map for socioeconomic classifications and average land-values, 

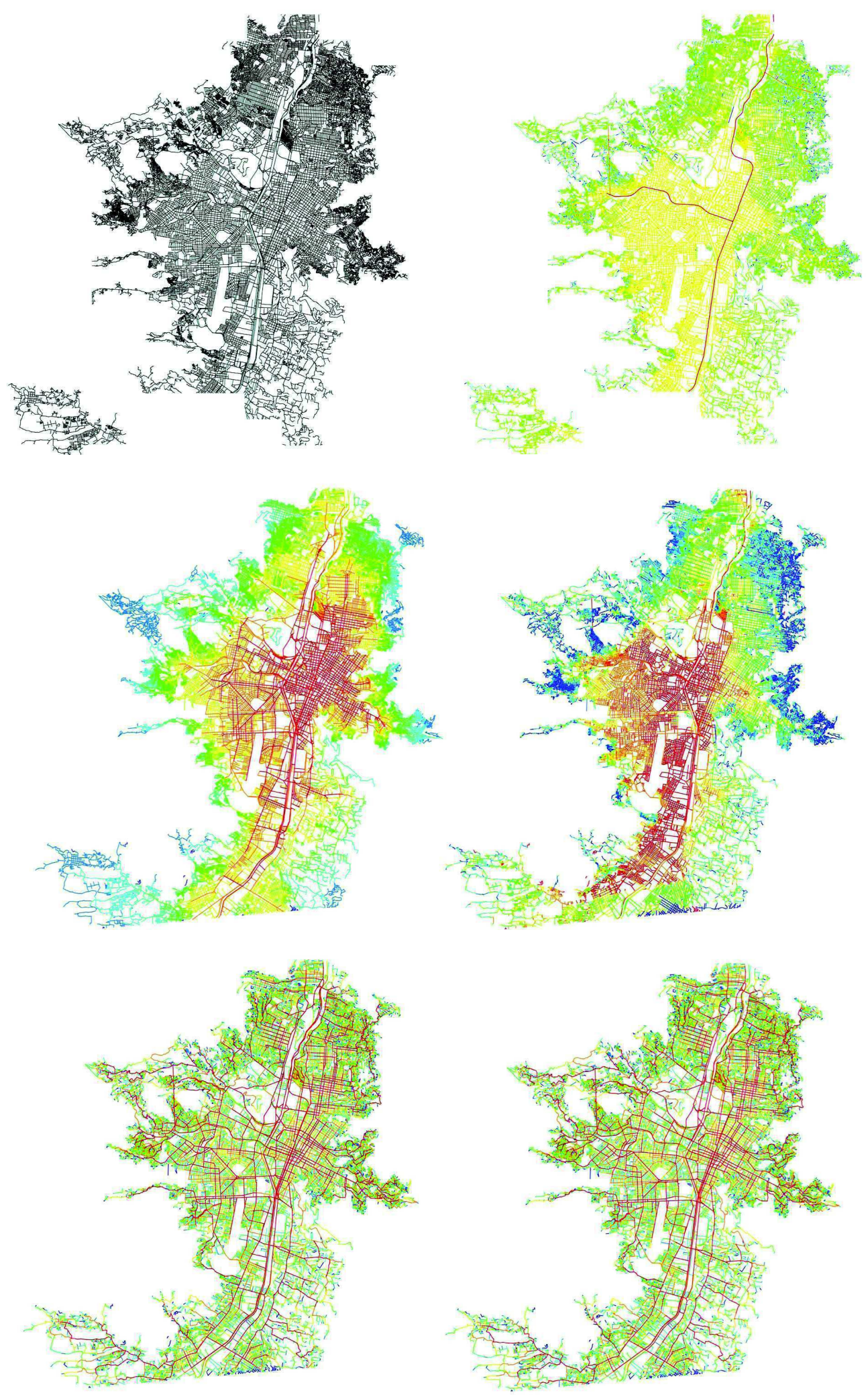

Figure 3.

Speed Maps. A) Medellin's urban grid un-analysed. B) Medellin speed map. C )Left - Standard Integration Map, Right - 'Speed-Integration' Map. D) Left - Standard Choice Map, Right - 'SpeedChoice' Map. 
suggesting it is possible to spatially link that the poorest and most deprived areas in Medellin with the steepest areas of the city, locations where affluent citizens would least like to live. Then, when this map is examined more closely around each cable-car station it is possible to observe some initial findings. Such as the roads that run perpendicular to the stations usually run along the flattest areas of each location and thus have the faster averages speeds, whereas the roads that run parallel to the cable-car line are much slower. Curiously these perpendicular roads are often picked up as important 'through' routes in the spatial analysis.

From this it is clear to observe that the segments surrounding each station have a significant number of low speed streets connecting to the cable-car. For example, at Santo Domingo along Calle 105 (Figure 1), which was picked up as an important 'through' space in the previous spatial analysis, the average speed is $1.7 \mathrm{~km}$ per hour, yet along the main routes of Carrera 31 and 32, which are perpendicular to the station, the average speed is $3.39 \mathrm{~km}$ per hour. This confirms that this method is picking up the large differences in movement previously demonstrated using GPS.

However, while the speed map shows basic urban information about the city, it would be difficult to say that this demonstrates anything that is not obvious to the general observer or is more advanced than a contour map. Yet, it represents a dataset that can be easily combined with the values previous generated using standard Space Syntax measurements, in an effort to examine if the accuracy of certain spatial connections within the city are improved (Figure 3).

When these values are combined it is possible to observe initial findings by visually analyzing the results. When the previous measurement of 'Integration' includes the weighted value of speed there is a change in the citywide map, with the main areas of centrality appearing to be smaller and more defined, with significant areas to the east and north of the city. This suggests that 'speed' provides a more accurate account of the whole city. However, at the local scale integration visually remains the same. When the same study is completed using the measurement 'Choice' there is very little impact to the overall city network of 'through' spaces, yet when the results are examined more closely around each station, the local 'through' space appears clearer. This is mainly observed between the metric radii of 1250 and 3000 , which represents the network most closely associated with walking.

Hence, the introduction of speed as a weighted variable starts to show previous floors in spatial analysis more accurately to local conditions. However, it is important to test the relationship of these results to real movement patterns and this will also provide an opportunity to establish whether or not the 'speed' variable has improved the spatial model's relationship with movement.

When the measurement of 'SpeedIntegration' is correlated to the movement patterns there is no significant changes in the correlations and this is understandable because only the large citywide maps show change. Hence, locally measured movement patterns are very unlikely to be altered. Yet, when the measurement of 'Speed-Choice' is correlated to the same movement patterns there is a general improvement with the correlations. However, the distribution of the highest and lowest correlations within the analysed metric radii network are all exactly the same. This show that whilst speed is improving the correlation coefficient values for each analysed network the actual finding in relations to the impact of the cable-car are largely unaltered (Figure 3).

These results reveal that when the 'speed' variable is added to the choice measurements 'through' space correlated better to local movement and this is understandable because the value of choice has traditionally been associated with local movement.

While this starts to demonstrate the worthiness of 'speed' it is useful to examine if the same pattern is observed between different cases, since each of these have different types of landscape. Hence the results for each cablecar line and station will be compared and this will be examined using just three different metric radii, which each represent a different urban scale (Serra and Pinho, 2013) and only the results for 'Choice' will be investigated, since they most closely represent movement. Starting with the comparison between each 


\section{CORRELATION BETWEEN SPACE AND MOVEMENT METRIC RADIUS 10000}

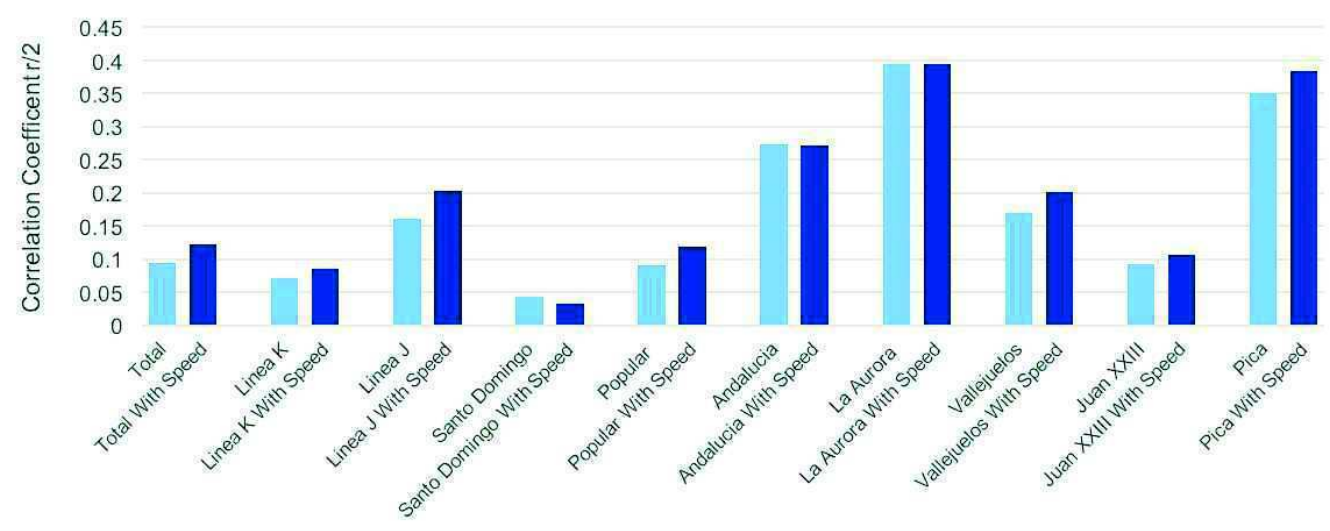

CORRELATION BETWEEN SPACE AND MOVEMENT

METRIC RADIUS 10000

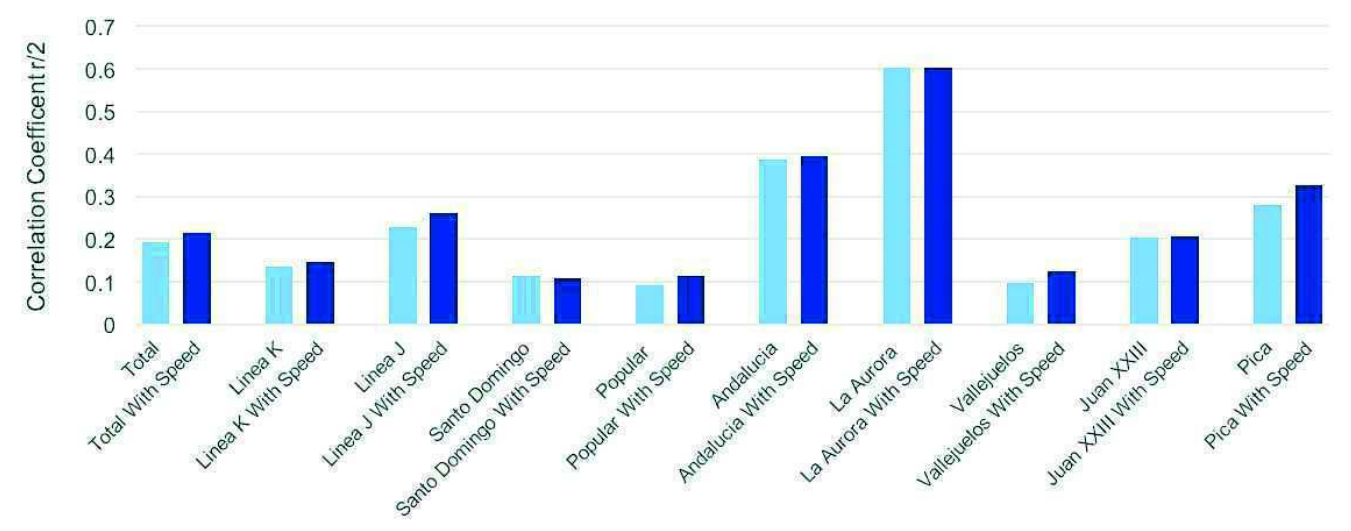

CORRELATION BETWEEN SPACE AND MOVEMENT METRIC RADIUS 10000

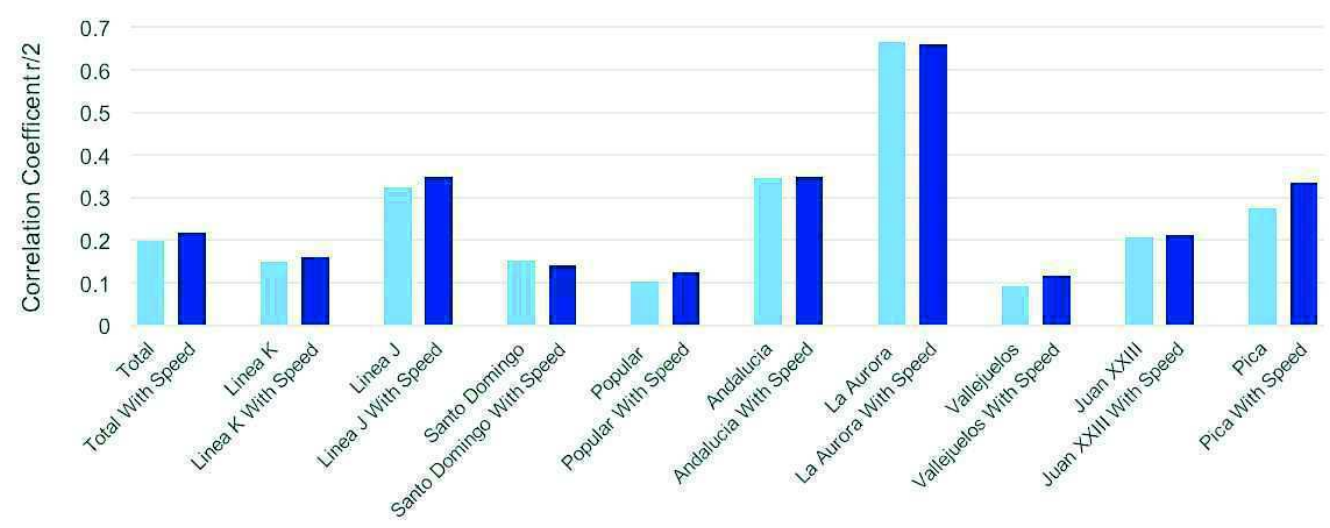

Figure 4.

Space and Movement. The correlation coefficient values between movement and space. Light Blue columns indicates standard spatial analysis; Dark Blue indicates 'Speed-Space' analysis. A) Metric Radius 1000. B) Metric Radius 3500. C) Metric Radius 10000. 
line both Linea $\mathrm{K}$ and Linea $\mathrm{J}$ the biggest improvements are at the metric radius 1000 with an increase between $15 \%$ and $21 \%$, whereas with the metric radii of both 3500 and 10000 the improvements are smaller between $12 \%$ and $7 \%$. This generally represents the same trend for all movements combined and as before the correlation coefficient values still remain low. This shows that the smallest network analysed, metric radius 1000, is improving the most and this is understandable since it is the most relatable network to walking, whereas the larger networks relate stronger to the road network (Figure 4).

However, when the surrounding area of each cable-car station is compared the results are more varied. For example, the stations along Linea $\mathrm{K}$ all show different results, at Santo Domingo the introduction of speed worsens the correlation at all three metric radii by between $-32 \%$ and $-6 \%$, yet at Popular it improves this by between $24 \%$ and $17 \%$ and at Andalucia there is virtually no difference. For the stations along Linea J, at La Aurora the changes are very minimal, then at Vallejuelos the differences are again significant being between $21 \%$ and $15 \%$ and at Juan XXIII again the metric radii show positive changes between $14 \%$ and $2 \%$. Similar to Linea K each case shows very different results, yet the landscape at each station along Linea $\mathrm{J}$ is also very different and as expected this should affect the results. This is because at La Aurora, which was recorded as the flattest case the results are virtually unchanged, whereas at both Vallejuelos and Juan XXII for metric radius $1000 \mathrm{~m}$, which represents a walking network, the changes are more significant. Then at Linea $\mathrm{K}$ the landscape is similar between each case, however the results are very different and this is most likely the effect of the larger attraction of the cable-cars. Here the cable-car is more
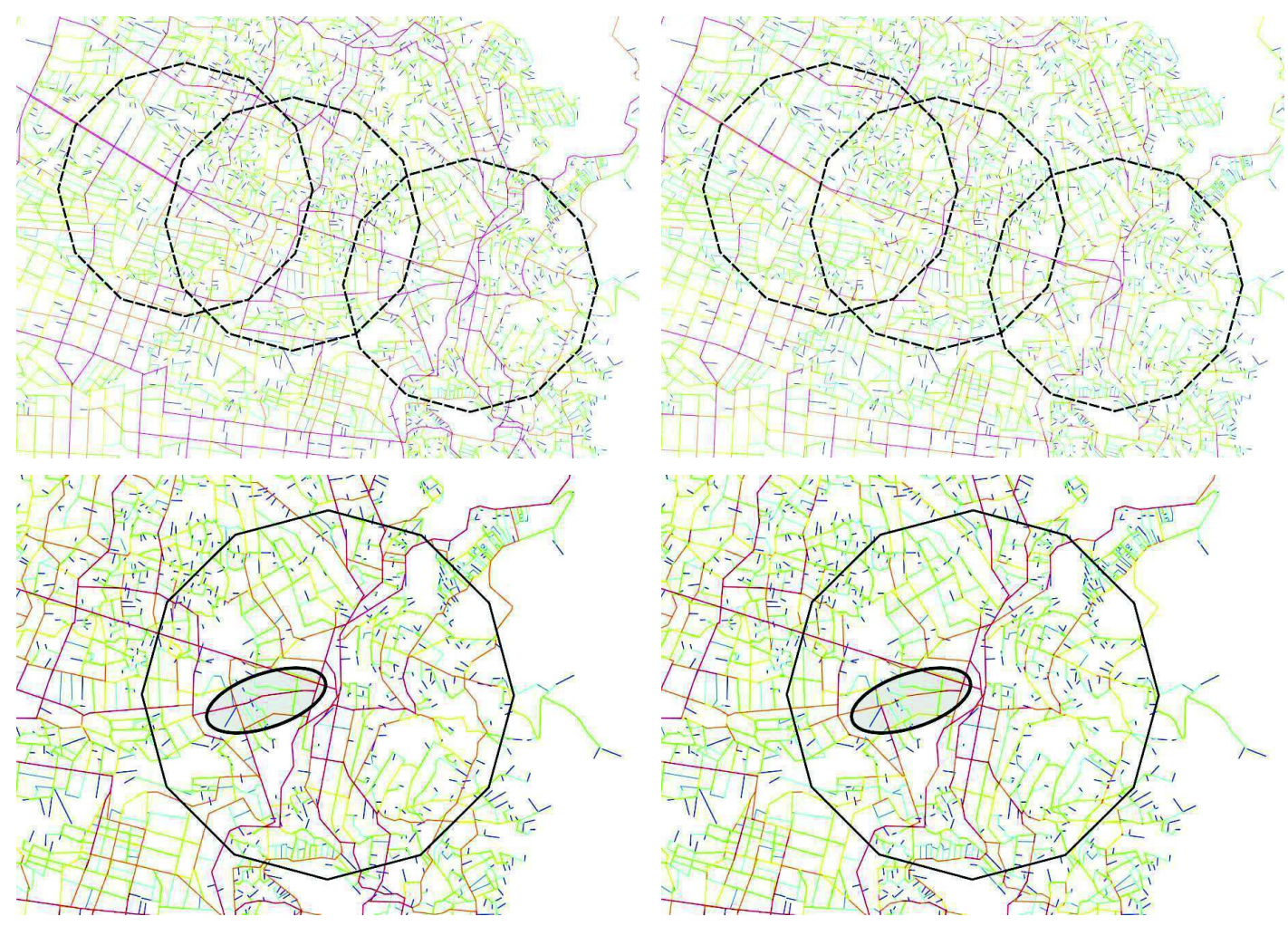

Figure 5.

Local Speed-Choice Results. A) Standard Choice at Metric Radius 3500 for area surrounding Linea K. Dashed circle indicates studied area for each cable-car station. B) 'Speed-Choice' at Metric Radius 3500 for area surrounding Linea K. C) Standard Choice at Metric Radius 3500 for area surrounding Santo Domingo. Highlighted circle indicates Calle 105 (figure 1). D) 'Speed-Choice' at Metric Radius 3500 for area surrounding Santo Domingo. Highlighted circle indicates Calle 105 (figure 1). 
heavily used, especially at Santo Domingo, this has the effect of encouraging a lot of movement towards each station no matter the condition of the pathway. Hence, at Santo Domingo the correlation worsens because the attraction of the cable-car and its nearby local commerce encourage pedestrian to use paths that have low spatial values. Whereas at Popular and Andalucia the cable-car's popularity is less, so movement is less likely to draw towards each station.

This can be further demonstrated by completing a similar study on a case that has a comparable landscape but no transport hub to attract movement. This is done using the case of Picacho on the opposite side of the valley to Linea K. Here the introduction of speed improves all three metric radii by between $8 \%$ and $18 \%$, similar to when all movement and each cable-car line is related to the speed-space network. It is also worth noting that unlike the other cases the correlation coefficient values here are reasonable between $0.3 \mathrm{r} / 2$ and $0.5 \mathrm{r} / 2$, both with and without 'speed'. This demonstrates how the relationship between space and movement is often skewed when there is a central point of attraction.

While the relationship between local movement and space can often be influenced by local attractors, when individual streets are observed that are known to be affected by landscape the exact impact of combining speed can be measured. When Calle 105 at Santo Domingo was previously examined it was shown to be significantly slower than other street in the neighbourhood, yet the spatial analysis indicated this street as being an important 'through' space. However, when the exact values for this street are examined with 'speed' its importance decrease as the spatial values generated drops by $37 \%$ at metric radius 1000 , and also at the other metric radii of 3500 and 10000 , by $9 \%$ and $18 \%$ respectively. Whereas the other street that was also examined Carrera 31 and 32, while there is also a drop at metric radius 1000 by $27 \%$, there is an increase in the values at both metric radii 3500 and 10000 , by $7 \%$ and $9 \%$ respectively. This starts to show that the streets that are most affected by the landscape see a decrease in the spatial values calculated and this is most noticeable at a walking scale (Figure 4).
So while the introduction of speed increases the correlation coefficient between space and movement within local areas the influence of 'speed' is even higher when individual streets are examined. Hence, its use starts to show a more localised representation of each local urban grid, yet when the impact of a spatial connection like the cable-car is sought this method does not alter the overall results.

\section{Findings}

By introducing speed and combining it with the previous space syntax analysis of Medellin the 'to' space become more reflective of the city as a whole and the local 'through' space was easier to interpret.

This is because the central integration zones are more central and to the west of the city and reduces the areas to the north and east of the city centre that were previously well integrated. This intuitively represents a better image of the main areas of centrality, as the areas to the north and east of the city centre are very steep and harder to walk. However, when the speedintegrations values for each individual case study is examined visually there are very few differences. So while speed is helping to clarify the integration values for the whole city, it is doing very little to improve the understanding of each individual case.

Yet, with the choice-speed measurements it becomes clear speed is enhancing the interpretation of natural 'through' spaces within the local areas surrounding each case study. Speed also makes local movement patterns easier tounderstand, as itdemonstrates how local natural movement can be better understood. In Santo Domingo, the introduction of speed decreases the importance of steep streets like Calle 105, yet it also increases the importance of streets like Carrera 31 (Figure 5). This starts to further suggest speed is helping to improve the understanding of natural movement. This is also noticeable at La Aurora, where the landscape is relatively flat in comparison to other stations, so the introduction of speed does little to improve correlations. Yet there are also local attractors that influence the relationship between movement and space as the cable-car stations and surrounding commerce encourage 
people to move towards them even if the roads are badly connected or very steep. This is less noticeable at cases like Picacho that do not have central points of attraction.

Yet, when the same measurements are observed at a city scale, the use of speed is less relevant, since none of the major through routes are altered. Therefore, the use of speed improves the integration results for the city's most central 'to' spaces, then with choice it makes local 'through' spaces, specifically where natural movement occurs, easier to understand.

While the combination of 'Speed' and 'Choice' improves the visual interpretation of the local areas and enhance the correlation with movement by between 10 and $15 \%$, when the distributions of the top and bottom correlations are observed these rarely alter. Therefore, if this is examining the impact of the cablecar and not the role of the landscape, the introduction of speed does very little to alter the findings, since the most significant metric radii with the highest correlation coefficient values are exactly the same as the standard spatial analysis. This demonstrates that the use of speed in understanding the overall role of the cable-car in the transformation process of each informal settlement is not greatly improved, since the same findings are available without this measurement.

For this one individual case, it is clear that speed offers a variable that can alter and enhance spatial analysis results. This is specifically useful for cities like Medellin where the landscape and urban fabric prevent homogenous spatial analysis, therefore movement patterns need to be observed differently and speed provides a good solution to alter a spatial model. With the introduction of a measurement like speed it is possible to make the city more dynamic for the individual and mode of transport (Fig 06). For example, if a candy floss seller is unable to use the cablecar and metro overground train, they are most likely to use the bus or walk, so with the use of speed the spatial analysis model can be adapted for that individual who has specific needs. However, the real benefit of introducing a 'speed' variable to the analysis of a city like Medellin, is that it starts to include the threedimensional element on the local landscape, which is hugely significant here and this helps to represent pedestrian movement in a clearer manner.

\section{Reflections}

The introduction of 'speed' creates a uniformed increase in the correlations between movement and space and this is also visually demonstrated in the spatial network of the city reflecting a more intuitive representation of the city. Yet there is little change in the distribution of the highest and lowest correlations for each analysed network, indicating that the measurable impact is not improved. Therefore, it is worthwhile reflecting on some of the elements that need refined for future research.

As the analysis includes a speed measurement for both metro-train and cable-car transport connections the most popular form of public transport in Medellin, the micro-buses are not included (Sarmiento et al., 2013). So while this form of transport is hard to map, as previously mentioned, it is still important to include this in future studies since it is an essential from of transport for this city. It is also worth noting that the speed of the public transport represented at average speed for the whole line, but this does not include the transition times through each stations or varying speeds for different parts of each line. This is important for the overground train, since it travels at different speed between different stations and waits longer at certain stations during rush hour, whereas the cablecar speed is fixed.

Another potential failing with testing the results is the movement survey. The speed weighted maps show clearly walking paths at a local scale and by tested this against the onsite observations these results should be verifiable, yet the movement survey was limited to 16 main junctions within $500 \mathrm{~m}$ radius of the cable-car station. This is because the movement surveys are a part of a research project that focusses on the impact of the cable-car and not testing 'speed' as a measurable variable. Hence, to improve accuracy future movement surveys should cover a larger area and include more pathways on steep inclines.

While the local movement survey and exact public transport speeds used to confirm the 


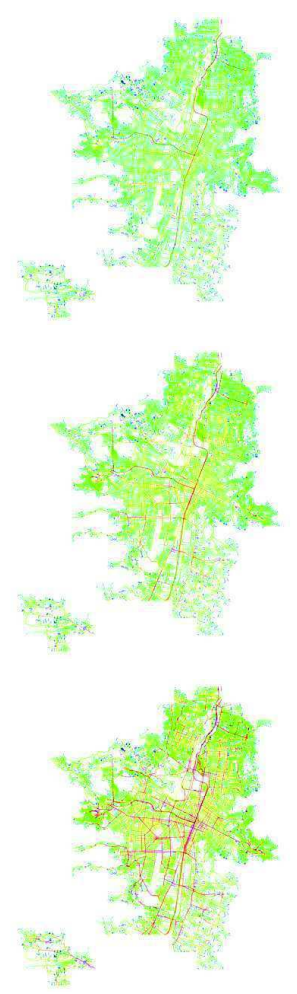

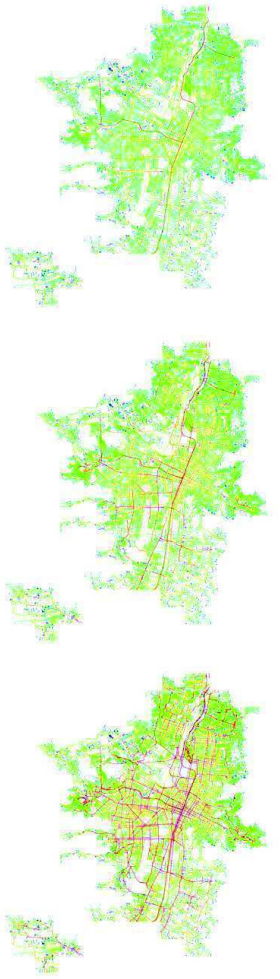

Figure 6.

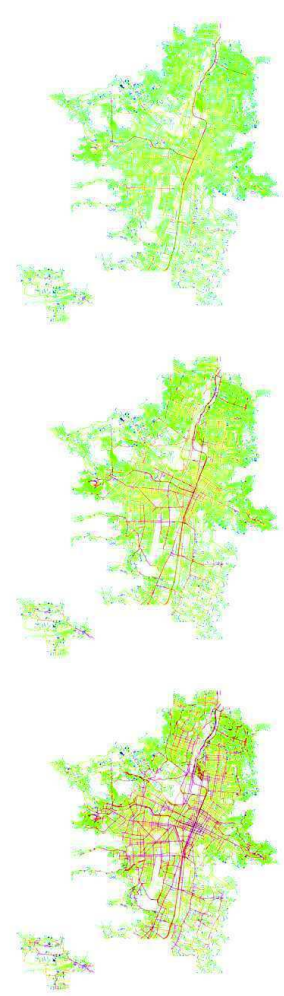

Dynamic Speed-Choice Potential. 'Speed-Choice' results using different average speeds. A) 'Speed-Choice' at $2 \mathrm{~km}$ per hour. B) 'Speed-Choice' at $3 \mathrm{~km}$ per hour. C) 'Speed-Choice' at $4 \mathrm{~km}$ per hour. D) 'Speed-Choice' at $5 \mathrm{~km}$ per hour. E) 'Speed-Choice' at $6 \mathrm{~km}$ per hour. F) 'Speed-Choice' at $7 \mathrm{~km}$ per hour. G) 'Speed-Choice' at $8 \mathrm{~km}$ per hour. H) 'Speed-Choice' at $9 \mathrm{~km}$ per hour. I) 'Speed-Choice' at $10 \mathrm{~km}$ per hour

results are not extensive enough to prove this is as a complete method, there is still enough evidence to suggest 'speed' could be fruitful for future spatial analysis. This is because the methods used here to formulate the speed map show clear merit and when combined with spatial analysis local improvements are observed. This could then provide a better opportunity to understand the spatial impact of different forms of movement. This is especially important when considering individual needs, as certain physical and social restriction often hamper movement, so the dynamic measurement of speed and mode of transport could be adapted for each individual case.

\section{Conclusion}

While this research strives to understand the impact of urban cable-cars, it is important to understand and evaluate the role that space has played in reconnecting isolated areas. One of the most reliable methods for doing this is Space Syntax. It has an established and trusted association with measuring and predicting the impact of spatial interventions and in the past has been used to discuss the transformation process of informal settlements. Yet with the case of Medellin, its steep sloping valleys force people to move differently making the use of Space Syntax questionable, since it does not traditionally pick up these local factors. It is for these reasons this paper has explored the worthiness of 'speed' in spatial analysis. It provides a pure value for the connection between different spaces representing the way individuals move along routes, whether steep or flat and accurately shows different forms of transport. So, by acknowledging that urban space is something more dynamic that simple linear connections, this provides an opportunity to explore how it can be perceived in different locations and through different mechanism that connect us from one space to another. 


\section{References}

Davila, J. (2013). Urban Mobility and Poverty: Lessons from Medellin and Soacha, Colombia.

Goodship, P., (2015). The impact of an urban cable-car transport system on the spatial configuration of an informal settlement.

Hillier, B. (2002). A theory of the city as object: or, how spatial laws mediate the social construction of urban space. Urban design international, 7(3-4), 153-179. doi: 10.1057/ palgrave.udi. 9000082

Hillier, B., Greene, M., \& Desyllas, J. (2000). Self-generated Neighbourhoods: the role of urban form in the consolidation of informal settlements. Urban design international, $5(2), 61-96$.

Hillier, B., (2007). Space is the machine: a configurational theory of architecture.

Karimi, K., Amir, A., \& Shaiei, K. (2007). Evidence-based spatial intervention for regeneration of informal settlements: the case of jeddah central unplanned areas.

Karimi, K., Parham, E., Friedrich, E., \& Ferguson, P. (2013). Origin-Destination Weighted Choice Model as a New Tool for Assessing the Impact of New Urban Developments. Paper presented at the Proceedings of the Ninth International Space Syntax Symposium, Seoul: Sejong University.

Law, S., Chiaradia, A., \& Schwander, C. (2012). TOWARDS A MULTI-MODAL SPACE SYNTAX ANALYSIS. A case study of the London street and underground network. Paper presented at the 8th International Space Syntax Symposium.

Liebst, L.S., (2015). Phenomenology of the movement economy: A multilevel analysis. The Journal of Space Syntax, 6(1), pp. $49-60$

Penn, A., (2003). The shape of habitable space.

McGuirk, J. (2014). Radical Cities: Across Latin America in Search of a New Architecture. London: Verso.

Netto, V.M.,(2015). "What is space syntax not?" Reflections on space syntax as sociospatial theory. Urban Design International, Vol. 21, 1, 25-40.

Ratti, C., (2004). Space syntax: some inconsistencies. Environment and Planning
B: Planning and Design, 31(4), pp.487-499.

Read, S., (2005). Flat city: a space syntax derived urban movement network model. In Proceedings of the 5th Space Syntax Symposium.

Segre, R. (2009). Formal-Informal Connections in the Favelas of Rio de Janeiro: The FavelaBairro Programme. Rethinking the Informal City: Critical Perspectives from Latin America, 11, 163.

Sarimiento, I., Cordoba, J., Mejia, A. \& Agudelo, L (2014). Metrocables and Travel Patterns in Medellin: The Inclusion of Latent Variable in Transport Models. [Chapter] Urban Mobility and Poverty: Lessons from Medellin and Soacha, Colombia, edited Davila, J.

Varoudis, T. (2012). 'depthmapX MultiPlatform Spatial Network Analysis Software', Version 0.30 OpenSource, http:// varoudis.github.io/depthmapX/

Vaughan, L. (2007). The spatial syntax of urban segregation. Progress in Planning, 67(3), 205294. doi: 10.1016/j.progress.2007.03.001

Vaughan, L., \& Arbaci, S. (2011). The challenges of understanding urban segregation. Built Environment, 37(2), 128138.

Vaughan, L.S. and Griffiths, S., 2013. A suburb is not a tree. Urban Design,125(Winter), pp.17-19.

UN-Habitat. (2013). State of the world's cities 2012/2013: prosperity of cities: Routledge.

Zampieri, F.L., Rigatti, D. and Ugalde, C., (2009). Evaluated model of pedestrian movement based on space syntax, performance measures and artificial neural nets. In Proceedings of the 7th international space syntax symposium (pp. 1-8). 